\title{
CT-Management des Lungenrundherds
}

\begin{abstract}
Lungenrundherde stellen immer ein diagnostisches Problem dar. Eine Studie aus den Niederlanden zeigt einen eleganten Weg auf, wie durch sequentielle CT-Volumetrie die Last der invasiven Diagnostik reduziert werden kann, ohne dass die diagnostische Sicherheit leidet.
\end{abstract}

- Zwischen April 2004 und Dezember 2006 rekrutierte man aus der niederländischen Bevölkerung 7557 Personen im Durchschnittsalter von $59 \pm 6$ Jahren mit einem medianen $\mathrm{Zi}$ garettenkonsum von $42 \pm 19 \mathrm{~Pa}$ ckungen pro Jahr zur Teilnahme an einer Screeninguntersuchung auf ein Bronchialkarzinom mithilfe Multi-Slice-Computertomografie. 16\% der Studienteilnehmer waren Frauen.

In dieser Gruppe entdeckte man bei 50,5\% insgesamt 8623 nicht kalzifizierte pulmonale Herde, von denen $98 \%$ sich als solide erwiesen. In der ersten Runde des Screenings wurden Patienten als negativ betrachtet, wenn das Rundherdvolumen weniger als $50 \mathrm{~mm}^{3}$ betrug, wenn es bei einem Volumen zwischen 50 und $500 \mathrm{~mm}^{3}$ bei einem Kontroll-CT nach drei Monaten nicht zugenommen hatte oder wenn im Fall eines Wachstums die Volumenverdopplungszeit 400 Tage oder mehr betrug. Unter diesen Prämissen war das Screeningergebnis nach der ersten Runde bei $79,2 \%$ negativ, bei $19,2 \%$ nicht eindeutig und bei $1,6 \%$ positiv.

Durchschnittlich $100 \pm 19$ Tage nach dem Ausgangs-CT erfolgte bei den Personen mit unklarem Ergebnis ein weiteres CT. Nach der ersten und zweiten Screeningrunde hatten 2,6\% bzw. 1,8\% der Studienteilnehmer ein positives Testergebnis. Von 196 Teilnehmern mit einem positiven Ergebnis konnten 177 weiter invasiv untersucht werden. Bei 70 dieser 177 Patienten ergab sich ein Bronchialkarzinom. Von den übrigen 107 Personen hatten 100 eine benigne Erkrankung und sieben Metastasen anderer Karzinome.

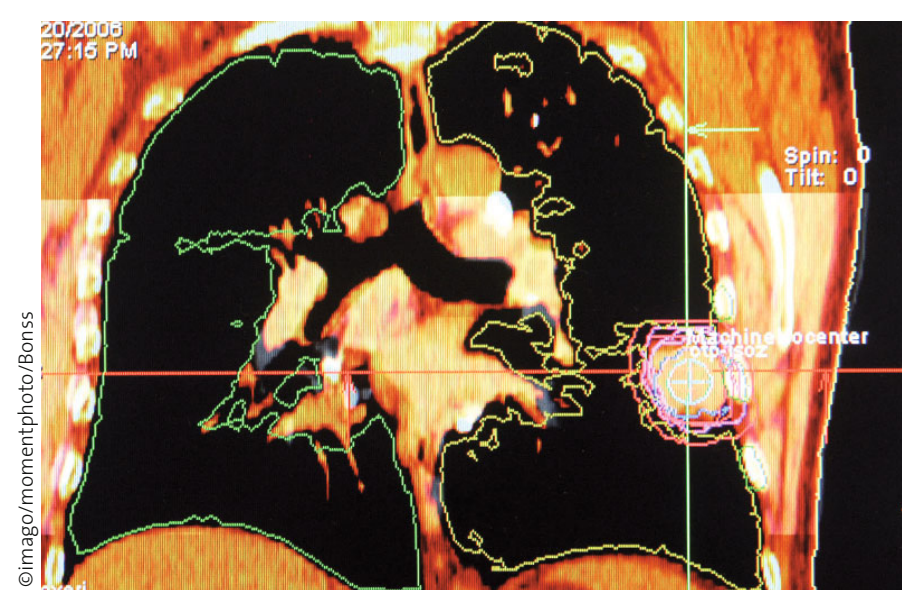

4 Lungenrundherd: Das Volumen verrät die Prognose.
Von 7361 Personen mit einem negativen Screeningergebnis in der ersten Runde entwickelten nur 20 in den darauf folgenden zwei Jahren ein Karzinom.

Die Studie ist auf zehn Jahre Nachbeobachtung hin angelegt, doch lässt sich bereits nach dem drei Jahre überblickenden Zwischenbericht sagen, dass mithilfe der genauen und sequentiell angewandten Volumetrie nach einem ersten negativen Testergebnis das Risiko für ein Bronchialkarzinom nach einem Jahr 1:1000 und nach zwei Jahren 3:1000 beträgt.

\section{Kommentar}

Mit dem Verfahren der Volumetrie unter exakt standardisierten Bedingungen lässt sich bei einer Hochrisikogruppe von Rauchern mit pulmonalen Rundherden die
Rate invasiver Untersuchungen erheblich reduzieren, ohne dass man diagnostische Sicherheit verliert. Allerdings sollte man nicht vergessen, dass es sich hier um eine systematische Studie handelt, an der eine kleine Gruppe von radiologischen Abteilungen teilnahm, die einen exakten Standard beachtete. Diese Voraussetzungen dürften in der üblichen Versorgungslandschaft, insbesondere in Deutschland, kaum gegeben sein. Hierzulande scheitert es oft schon daran, dass Voraufnahmen nur mit einem erheblichen organisatorischen Aufwand zu bekommen sind und der technische Standard durchaus heterogen ist.

H. S. FüEßL =

\footnotetext{
- R. V. van Klaveren et al.

Management of lung nodules detected by volume CT scanning. New Engl. J. Med. 361
} (2009) 23, 2221-2229 